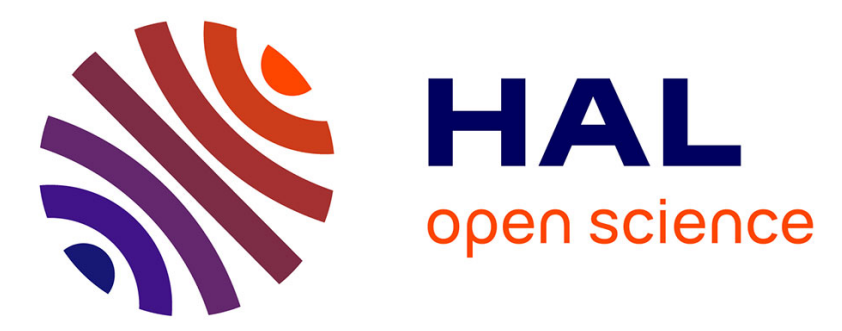

\title{
Pareamento de Subportadoras com Redução de PAPR em Sistemas OFDM Cooperativos
}

\author{
Pedro Marinho R. de Oliveira, C. Alexandre Rolim Fernandes
}

\section{To cite this version:}

Pedro Marinho R. de Oliveira, C. Alexandre Rolim Fernandes. Pareamento de Subportadoras com Redução de PAPR em Sistemas OFDM Cooperativos. XXXIV Simpósio Brasileiro de Telecomunicações e Processamento de Sinais, Aug 2016, Santarém, Brazil. 10.14209/sbrt.2016.38 hal-01954425

\section{HAL Id: hal-01954425 \\ https://hal.science/hal-01954425}

Submitted on 13 Dec 2018

HAL is a multi-disciplinary open access archive for the deposit and dissemination of scientific research documents, whether they are published or not. The documents may come from teaching and research institutions in France or abroad, or from public or private research centers.
L'archive ouverte pluridisciplinaire HAL, est destinée au dépôt et à la diffusion de documents scientifiques de niveau recherche, publiés ou non, émanant des établissements d'enseignement et de recherche français ou étrangers, des laboratoires publics ou privés. 


\title{
Pareamento de Subportadoras com Redução de PAPR em Sistemas OFDM Cooperativos
}

\author{
Pedro Marinho Ramos de Oliveira e C. Alexandre Rolim Fernandes
}

\begin{abstract}
Resumo-Neste artigo, propõe-se duas novas técnicas de redução de PAPR (Peak-to-Average Power Ratio) em sistemas de comunicação cooperativos OFDM (Orthogonal Frequency Division Multiplexing) utilizando o protocolo de transmissão fixo AF (Amplify-and-Forward). As técnicas propostas são baseadas em métodos de pareamento de subportadoras. $O$ desempenho das técnicas propostas serão avaliadas por meio de simulações computacionais, comprovando a eficiência das mesmas tanto na redução da PAPR no relay, quanto na SER (Symbol Error Rate) e na capacidade do sistema.
\end{abstract}

Palavras-Chave-PAPR, OFDM, Comunicação Cooperativa, Pareamento de Subportadoras.

Abstract-This paper proposes two new techniques of PAPR reduction for cooperative OFDM communication systems using the fixed AF transmission protocol, the proposed techniques being based on subcarrier pairing methods. The performance of the proposed techniques is evaluated by means of computational simulations, showing the efficiency of the methods in reducing the PAPR at the relay, as well as in providing good SER and capacity.

Keywords-PAPR, OFDM, Cooperative Communication, Subcarrier Pairing.

\section{INTRODUÇÃO}

Com o objetivo de fornecer um aumento significativo na potência recebida e uma melhor qualidade do sinal, foi proposto o conceito de sistemas de comunicações cooperativas, em que usa-se pelo menos uma antena retransmissora chamada relay para ajudar na comunicação entre a fonte e o destino [1]. Uma comunicação cooperativa pode ser feita por meio de diversos protocolos cooperativos, que podem ser classificados como fixos ou adaptativos. Devido à sua simplicidade, no presente trabalho será considerado o protocolo fixo AF (Amplifyand-Forward), em que o relay amplifica o sinal recebido da fonte e retransmite para o destino.

Por outro lado, com o objetivo de melhor combater a seletividade em frequência dos canais de comunicação rádiomóveis, foram criadas as técnicas de transmissão multiportadora, em que pode-se destacar a OFDM (Orthogonal Frequency Division Multiplexing), bastante usada atualmente nos sistemas de comunicações móveis da quarta geração [2]. Dentre as vantagens que a OFDM oferece, vale a pena citar a eficiência espectral, o combate a seletividade em frequência e a simplicidade de implementação.

Uma das principais desvantagens dos sistemas OFDM são os altos valores de PAPR (Peak-to-Average Power Ratio), que

Pedro Marinho Ramos de Oliveira e C. Alexandre Rolim Fernandes, Departamento de Engenharia da Computação, Universidade Federal do Ceará, Sobral-CE, Brasil, E-mails: pedromarinh8@gmail.com, alexandre_ufc@yahoo.com.br. C. Alexandre Rolim Fernandes é parcialmente financiado pelo CNPq. é a razão entre a potência de pico e a potência média do sinal. Altos valores de PAPR fazem com que os sinais com altas amplitudes caiam na região não linear de amplificadores de potência e de outros dispositivos não lineares, podendo causar erros na detecção desses sinais [3].

Devido a esse problema, diversas técnicas de redução e controle de PAPR foram desenvolvidas para os sistemas ODFM de comunicações móveis, como a SLM (Selective Mapping) [4] e a PTS (Partial Transmission Sequence) [5]. Algumas variações da técnica PTS foram propostas, como a Cooperative PTS em [6], PTS para sistemas MIMO (Multiple Input Multiple Output) OFDM [7] e a PTS-Modificado [8], que consiste em juntar as técnicas SLM e PTS citadas anteriormente. Em [9], foi proposta uma técnica de redução de PAPR utilizando compressões e expansões polinomiais, no transmissor e no receptor, respectivamente. Apesar de muito se ter feito para sistemas OFDM, pouco se tem feito em relação a esse problema para o caso específico de sistemas OFDM cooperativos AF. De fato, alguns poucos trabalhos exploraram as particularidades de um sistema cooperativo para se reduzir a PAPR em um relay $\mathrm{AF}$.

No cenário cooperativo foram propostas algumas técnicas, como em [10] para sistemas cooperativos VMIMO (Virtual MIMO) OFDM utilizando o protocolo AF e em [11], para sistemas cooperativos SFBC (Space-Frequency Block Coded) OFDM. Em [12], foi proposta uma técnica de redução de PAPR no relay utilizando o pareamento das subportadoras do link SR (Source-Relay) com as subportadoras do link RD (Relay-Destination).

$\mathrm{O}$ pareamento de subportadoras consiste em transmitir o símbolo que foi transmitido em determinada subportadora do link SR em determinada subportadora do link RD, formando assim pares de subportadoras [12]. Vale citar algumas técnicas de pareamento de subportadoras, como a BTB (Best-To-Best) [13], em que a melhor subportadora do link SR é pareada com a melhor subportadora do link RD, e a BTW (Best-To-Worst) [13], que faz o pareamento da melhor subportadora do link SR com a pior subportadora do link RD.

Devido aos seus benefícios em oferecer uma baixa SER (Symbol Error Rate), a técnica de pareamento de subportadora BTW foi utilizada no trabalho [12] como base para a proposição de uma técnica de pareamento de subportadoras com redução de PAPR em sistemas OFDM cooperativos AF. A técnica proposta em [12], chamada de Shifted BTW (SBTW), visa obter um baixo valor de PAPR à medida que também fornece uma SER aceitável, utilizando pareamento de subportadoras combinado com uma técnica de redução de PAPR.

Com base nesse cenário, o presente trabalho apresenta 
duas novas técnicas de redução de PAPR no relay utilizando pareamento das subportadoras dos links de comunicação cooperativos usando o protocolo AF, considerando um enlace descendente. A primeira das técnicas propostas, denominada Modified Shifted BTW (MSBTW), é uma modificação da técnica SBTW que calcula o valor da PAPR para diversos pareamentos, para então selecionar aquele com o menor valor de PAPR.

A segunda técnica proposta, denominada Source Implemented Modified Shifted BTW (SI-MSBTW), é uma versão da MSBTW em que todos os cálculos são realizados na fonte, utilizando uma estimação não ruidosa do sinal transmitido pelo relay. A técnica SI-MSBTW remove a complexidade no relay e a desloca para a fonte. Isto é desejável em um enlace descendente, uma vez que a fonte é a estação-base e o relay é uma unidade móvel ou pequena estação retransmissora.

As técnicas propostas serão avaliadas por meio de simulações computacionais, comparando-as com a técnica SBTW, proposta em [12], bem como com a técnica de pareamento BTW.

O restante deste artigo está dividido da seguinte forma. A Seção II apresenta o modelo do sistema cooperativo usado ao longo do presente trabalho. Na Seção III é apresentada com mais detalhes a técnica proposta em [12], bem como as novas técnicas propostas. A Seção IV apresenta os resultados das simulações computacionais. Por fim, a Seção V apresenta as conclusões do presente trabalho, bem como as perspectivas de trabalhos futuros.

\section{Modelo de Sistema}

Considera-se um sistema de comunicação cooperativo OFDM com $N_{s}$ subportadoras usando apenas uma fonte, um único relay operando com o protocolo AF fixo e um destino, como ilustrado na Figura 1. Todos os nós estão equipados com uma única antena operando em modo half-duplex e os canais possuem desvanecimento Rayleigh, sendo independentes e identicamente distribuídos. Os sinais recebidos tanto no relay quanto no destino presenciam um ruído do tipo AWGN (Additive White Gaussian Noise) de média nula. Assumese que os símbolos de informação transmitidos são do tipo QAM (Quadrature Amplitude Modulation) ou PSK (Phase Shift Keying) uniformemente distribuídos. Ao chegarem no destino, os sinais oriundos da fonte e do relay são combinados por um receptor MRC (Maximal Ratio Combining).

Considera-se também que o sinal OFDM é amplificado por um amplificador de potência (PA, do inglês Power Amplifier) não linear no relay, enquanto que a fonte possui um PA linear. Essa hipótese é feita pois é considerado um enlace descendente, em que a fonte é uma estação base com um PA de maior qualidade, enquanto que o relay é uma unidade móvel ou uma pequena estação base com menor recursos de hardware.

Desse modo, o sinal recebido pela $n$-ésima subportadora no relay, no domínio da frequência, é dado por:

$$
Y_{n}^{S R}=\sqrt{P_{s}} H_{n}^{S R} X_{n}+N_{n}^{S R},
$$

em que $H_{n}^{S R}$ e $N_{n}^{S R}$ são a resposta em frequência do canal e o ruído no domínio da frequência, respectivamente, na $n$ -

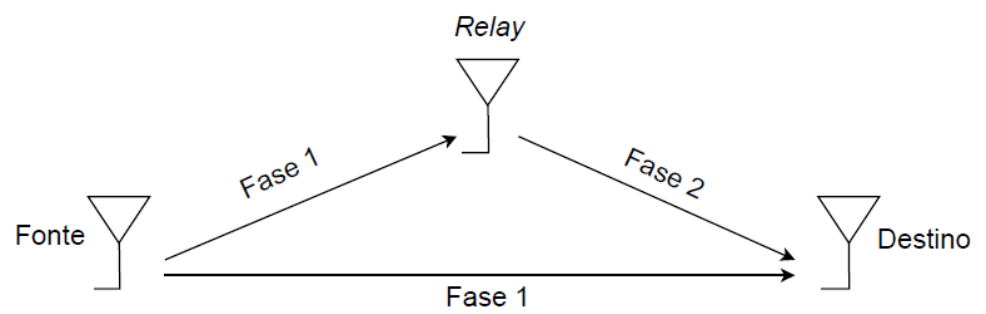

Fig. 1. Modelo de Sistema de Comunicação OFDM Cooperativa.

ésima subportadora do link $\mathrm{SR}, P_{s}$ é a potência na fonte e $X_{n}$ é o sinal de informação. Assumindo que o relay possui conhecimento do canal SR, o sinal $Y_{n}^{S R}$ é amplificado no domínio da frequência pelo relay utilizando-se o seguinte ganho variável:

$$
\beta_{n}=\frac{\sqrt{P_{r}}}{\sqrt{P_{s}\left|H_{n}^{S R}\right|^{2}+P_{n}}},
$$

em que $P_{r}$ e $P_{n}$ são as potências do relay e do AWGN no link entre a fonte e o relay, respectivamente.

O pareamento das subportadoras é realizado logo após a amplificação no relay, com o intuito de melhorar o desempenho do sistema em termos de SER e/ou capacidade. As técnicas de pareamento das subportadoras são detalhadas na Seção III. Assumindo que a $n$-ésima subportadora do link SR é associada com a $m$-ésima subportadora do link $\mathrm{RD}$, temos que o sinal oriundo do relay recebido pela $n$-ésima subportadora no destino é dado por:

$$
Y_{n}^{R D}=H_{m}^{R D} \mathcal{F}_{n}\left(\mathbf{f}\left(\mathbf{y}^{S R-R D}\right)\right)+N_{m}^{R D}
$$

em que $H_{m}^{R D}$ e $N_{m}^{R D}$ são o canal e o ruído representados no domínio da frequência, respectivamente, na $m$-ésima subportadora do link $\mathrm{RD}, \mathcal{F}_{n}(\cdot): C^{N} \rightarrow C$ é a função que retorna a $n$-ésima subportadora da transformada discreta de Fourier do vetor de entrada, $\mathbf{f}(\cdot): C^{N} \rightarrow C^{N}$ é a função que modela o amplificador de potência não linear no relay e $\mathbf{y}^{S R-R D} \in C^{N}$ é um vetor que contém os sinais $y_{n^{\prime}}^{S R-R D}$ transmitidos pelo relay no domínio do tempo, sendo $n^{\prime}$ a variável que representa o tempo discreto. $\mathrm{O}$ sinal $y_{n^{\prime}}^{S R-R D}$, por sua vez, é a transformada discreta de Fourier inversa do sinal $\beta_{n} Y_{n}^{S R-R D}$, sendo $Y_{n}^{S R-R D}$ o sinal $Y_{n}^{S R}$ já pareado.

$\mathrm{O}$ sinal oriundo da fonte recebido pela $n$-ésima subportadora no destino é dado por

$$
Y_{n}^{S D}=\sqrt{P_{s}} H_{n}^{S D} X_{n}+N_{n}^{S D},
$$

em que $H_{n}^{S D}$ e $N_{n}^{S D}$ correspondem a resposta em frequência do canal e o ruído no domínio da frequência, respectivamente, na $n$-ésima subportadora do link SD (Source-Destination). $\mathrm{O}$ sinal combinado no destino pelo receptor MRC pode ser calculado como $Y=a_{1} Y^{S D}+a_{2} Y^{R D}$, em que assume-se conhecidos os coeficientes $a_{1}$ e $a_{2}$.

A PAPR, definida como a razão entre a potência de pico e a potência média do sinal, é dada em $\mathrm{dB}$ no relay por:

$$
P A P R_{r}=10 \log _{10}\left[\frac{\max _{1 \leq n^{\prime} \leq \tilde{N}}\left[\left|y_{n^{\prime}}^{S R-R D}\right|^{2}\right]}{E\left[\left|y_{n^{\prime}}^{S R-R D}\right|^{2}\right]}\right],
$$




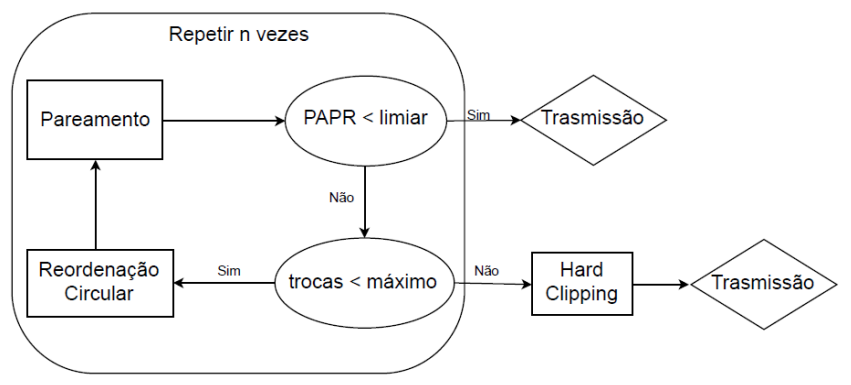

Fig. 2. Diagrama de blocos da técnica SBTW [12].

em que $E[\cdot]$ denota o operador esperança, $\max [\cdot]$ é a função que retorna o valor máximo e $y_{n^{\prime}}^{S R-R D}$ é o sinal transmitido pelo relay no domínio do tempo, após feito o pareamento das subportadoras.

A capacidade do sistema cooperativo AF é dada por

$$
C_{A F}=\sum_{n=1}^{N_{s}} C_{n}^{(A F)},
$$

em que $C_{n}^{(A F)}$ é a capacidade da $n$-ésima subportadora, dada por:

$$
C_{n}^{(A F)}=\frac{1}{2} \frac{1}{N_{s}} \log _{2}\left[1+\gamma_{1, n}+\frac{\gamma_{2, n} \gamma_{3, n}}{1+\gamma_{2, n}+\gamma_{3, n}}\right],
$$

sendo $\gamma_{1, n}, \gamma_{2, n}$ e $\gamma_{3, n}$ as SNRs instantâneas da $n$-ésima subportadora dos enlaces SD, SR e RD, respectivamente.

Alguns trabalhos demonstraram que, sob certas circunstâncias, a capacidade de sistemas OFDM cooperativos com PAs não lineares pode sofrer penalidades, assumindo valores menores que aqueles indicados em (7) [3]. Entretanto, estes resultados não consideram que nenhum tipo de técnica de redução de PAPR é aplicada nos sinais transmitidos. Desta forma, nas simulações computacionais neste trabalho, consideraremos que a capacidade é dada pela expressão (7).

\section{TÉCNICAS DE REDUÇÃo DE PAPR BASEADAS EM PAREAMENTO DE SUbPORTADORAS}

Nesta seção são apresentadas as técnicas de redução de PAPR baseadas em pareamento de subportadoras. Inicialmente, será apresentada a técnica SBTW, proposta em [12], e, em seguida, serão apresentadas as técnicas propostas. As técnicas discutidas a seguir supõem que o relay possui um conhecimento dos canais SR e RD.

\section{A. Shifted BTW (SBTW)}

A técnica SBTW consiste em, após aplicada a técnica BTW, em que a melhor subportadora de um enlace é pareada com a pior subportadora do outro enlace, calcular e comparar a PAPR do sinal com um limiar pré-determinado de PAPR. Caso a PAPR seja menor que esse limiar, o sinal é transmitido normalmente. Caso esse valor de PAPR medido seja maior que esse limiar, as subportadoras são reordenadas circularmente e um novo valor da PAPR é calculado e comparado novamente com o limiar de PAPR. Se após atingir um número máximo de reordenamentos (shifts) um valor da PAPR aceitável não for atingido, a amplitude do sinal é ceifada e nenhum reordenamento de subportadoras é realizado, ou seja, utiliza-se a técnica BTW como pareamento de subportadoras. Reordenar circularmente as subportadoras corresponde a trocar a ordem em que as subportadoras estão representadas no vetor de maneira circular. Por exemplo, a subportadora na posição 1 irá para a posição 2 , esta última para a posição 3 e assim por diante, até que a última subportadora fique na posição 1 .

Percebe-se que a técnica SBTW combina o pareamento de subportadoras com uma técnica de redução de PAPR. A Figura 2 ilustra o funcionamento dessa técnica através de um diagrama de blocos. A técnica SBTW mostrou-se capaz de diminuir a PAPR do sinal no relay [12], entretanto, ela possui algumas desvantagens. Uma destas desvantagens reside no fato de não minimizar a PAPR, ela apenas procura por um valor "aceitável" de PAPR. Neste caso, considera-se um valor aceitável de PAPR como sendo qualquer valor abaixo da PAPR limiar. Entretanto, mesmo os valores aceitáveis de PAPR utilizados em [12] podem levar a distorções importantes de PAPR. Ademais, quando o número máximo de reordenamentos é atingido, o sinal é distorcido ao ter sua amplitude ceifada, o que causa um aumento da SER.

Na próxima seção é apresentada uma técnica de redução de PAPR que minimiza estas desvantagens da técnica Shifted BTW.

\section{B. Técnica Proposta I: Modified Shifted BTW (MSBTW)}

A técnica proposta nesta seção, denominada Modified Shifted BTW, consiste em realizar o pareamento BTW, em seguida, calcular a PAPR do sinal, armazená-la e realizar o reordenamento circular, pareando as subportadoras novamente. Esse processo é feito um número pré-determinado de vezes, para que ao fim, um seletor selecione o pareamento que fornece o sinal com a melhor PAPR. Esse pareamento é então realizado e o sinal é transmitido para o destino. Esta técnica proposta é ilustrada pela Figura 3, através de um diagrama de blocos.

A vantagem deste método em relação à técnica Shifted BTW é que ela escolhe a menor PAPR dentre os valores calculados, ou seja, ela minimiza a PAPR dentro do conjunto de escolha. Neste caso, existe uma menor probabilidade de a PAPR levar à introdução de importantes distorções não lineares. Além disso, como será visto na seção de resultados de simulação, a técnica proposta não apresenta perdas em termos de capacidade. Desta forma, quando um modelo não linear de PA é considerado para o relay, a técnica proposta deve fornecer ganhos de SER em relação à técnica Shifted BTW.

$\mathrm{Na}$ próxima seção será apresentada uma segunda técnica proposta, cuja diferença em relação ao método Modified Shifted BTW consiste no fato de ela ser implementada pelo nó fonte.

\section{Técnica Proposta II: Source Implemented Modified Shifted BTW (SI-MSBTW)}

A segunda técnica proposta é uma versão da MSBTW em que todos os cálculos são realizados na fonte, utilizando uma estimação não ruidosa do sinal transmitido pelo relay. Em 


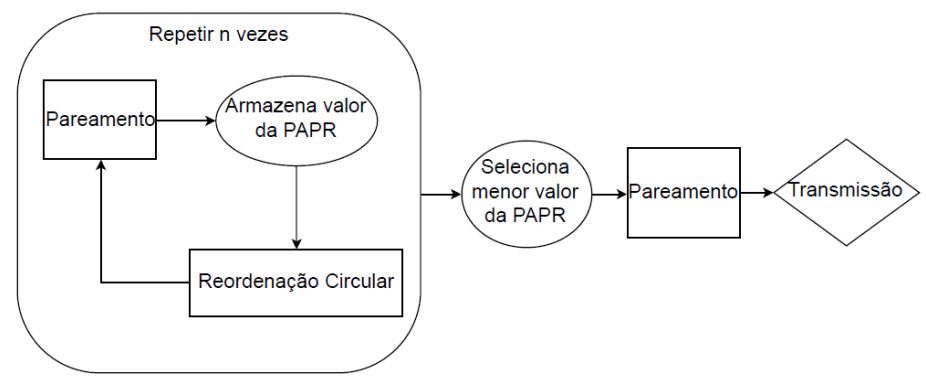

Fig. 3. Diagrama de blocos da técnica MSBTW.

TABELA I

ParÂMEtros usados nas SimulaÇões COMPUtacionais.

\begin{tabular}{|c|c|}
\hline $\mathrm{N}^{\circ}$ de Subportadoras & 256 \\
\hline$P_{s}$ & 0.5 \\
\hline$P_{r}$ & 0.5 \\
\hline $\mathrm{N}^{\circ}$ de Amostras de Monte Carlo & 10000 \\
\hline Tamanho do Prefíxo Cíclico & 3 \\
\hline $\mathrm{N}^{\circ}$ de Símbolos & 30 \\
\hline Ordem da Modulação QAM & 16 \\
\hline Limiar de Capacidade de Outage & 2 \\
\hline
\end{tabular}

outras palavras, este método consiste em, na fonte, estimar o sinal transmitido no relay, tomando como conhecidos os ganhos do canal SR, realizar os pareamentos utilizando este sinal estimado e por fim, selecionar o pareamento que fornece o menor valor de PAPR e informá-lo ao relay. Deste modo, quando o sinal oriundo da fonte chegar no relay, apenas o pareamento que fornece o menor valor de PAPR será realizado. A versão não ruidosa do sinal transmitido pelo relay estimada na fonte, no domínio da freqüência, é dada por: $Y_{n}^{e}=\beta_{n} X_{n} H_{n}^{S R}$

Esta técnica retira a complexidade no relay, deixandoa na fonte, já que o "melhor" pareamento já é conhecido quando o sinal chega no relay. Isto é desejável em um enlace descendente, uma vez que a fonte é a estação-base e o relay é uma unidade móvel ou uma pequena estação retransmissora.

\section{Resultados de Simulação}

Nesta seção são apresentados os resultados das simulações computacionais com o objetivo de avaliar o desempenho das técnicas propostas. As métricas de desempenho utilizadas são a CCDF (Complementary Cumulative Distribution Function) da PAPR no relay, a SER e a probabilidade de outage (probabilidade de interrupção), que é a probabilidade de a capacidade do sistema estar abaixo de um limiar pré-determinado. Para todas as simulações, foram considerados os parâmetros mostrados na Tabela I. Além disso, foi assumido que todos os canais possuem desvanecimento Rayleigh seletivo em freqüência com um espalhamento de atrasos de 3 taps. Neste trabalho será utilizado o modelo de PA não linear Soft Clipping, com amplitude de saturação igual a 1 [3].

A Figura 4 apresenta a CCDF da PAPR no relay usando a técnica de pareamento BTW, Shifted BTW e Modified Shifted BTW. Podemos observar nesta figura uma considerável diferença nas PAPRs da técnica de pareamento convencional BTW (que não busca diminuir a PAPR) para as PAPRs da

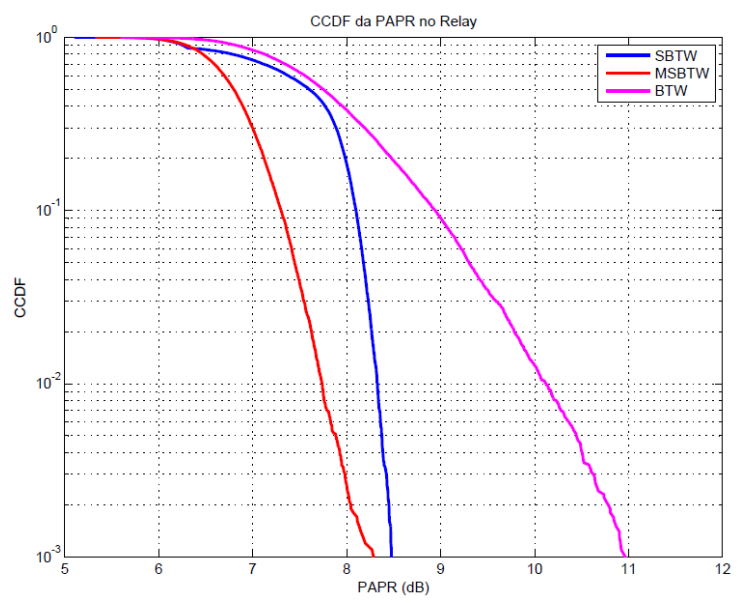

Fig. 4. CCDF da PAPR no relay para as técnicas de pareamento BTW, SBTW e MSBTW.

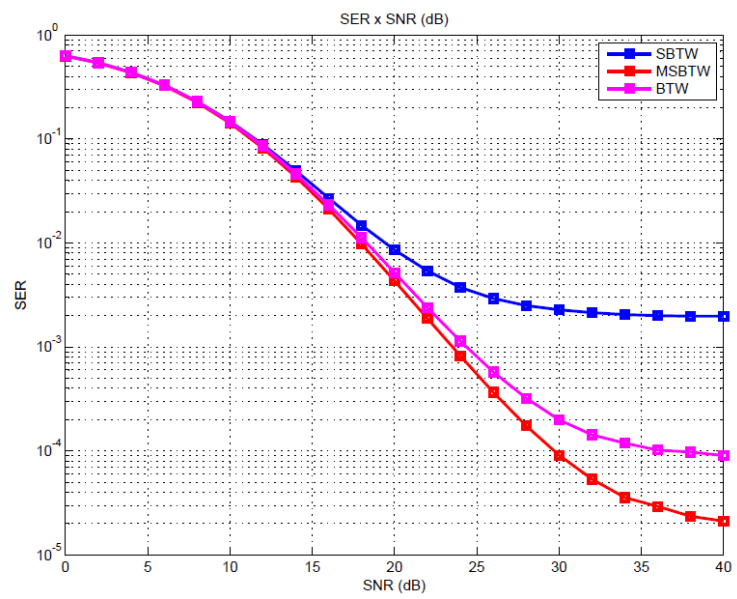

Fig. 5. SER versus SNR para as técnicas de pareamento BTW, SBTW e MSBTW.

técnicas Modified Shifted BTW e da Shifted BTW. Observase também que a técnica proposta neste trabalho oferece uma redução significativa da PAPR em relação à técnica Shifted BTW, pelos motivos anteriormente expostos.

A Figura 5 apresenta a SER em função da SNR média do sinal para as técnicas citadas anteriormente. Observa-se também que a técnica proposta MSBTW oferece uma menor SER, convergindo a um piso menor, quando comparada com as demais. Isto se deve à maior redução de PAPR obtida por esta técnica, tal como ilustrado na Fig. 4.

A Figura 6 apresenta a probabilidade de outage em função da SNR média para as técnicas citadas anteriormente. Pode-se observar que a técnica proposta no presente trabalho fornece uma probabilidade de outage equivalente às demais técnicas. Isto ocorre devido ao fato de os reordenamentos circulares dos pareamentos não afetarem de forma significativa a capacidade, fazendo assim com que as técnicas testadas tenham capacidades similares.

As Figuras 7 e 8 mostram, respectivamente, a probabilidade de outage e a SER para as duas técnicas propostas: MSBTW e SI-MSBTW. Pode-se observar que a técnica SI-MSBTW fornece uma probabilidade de outage e uma SER bastante 


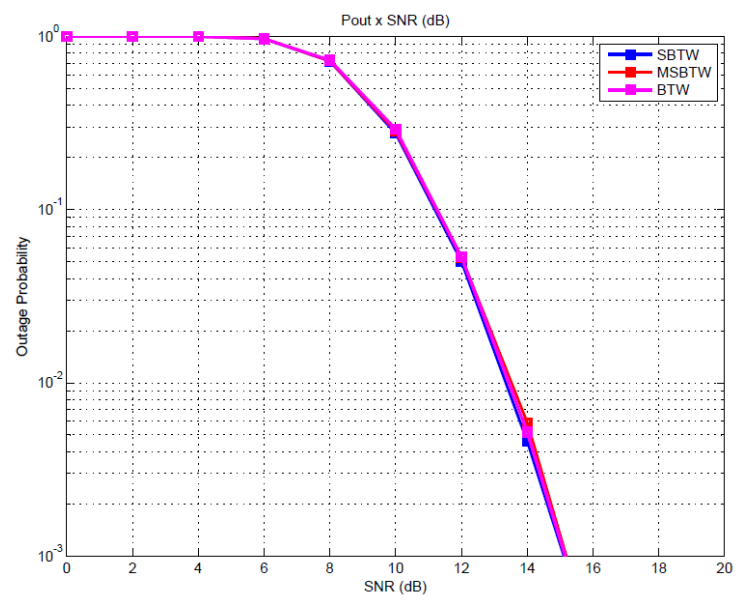

Fig. 6. Probabilidade de outage versus SNR para as técnicas de pareamento BTW, SBTW e MSBTW.

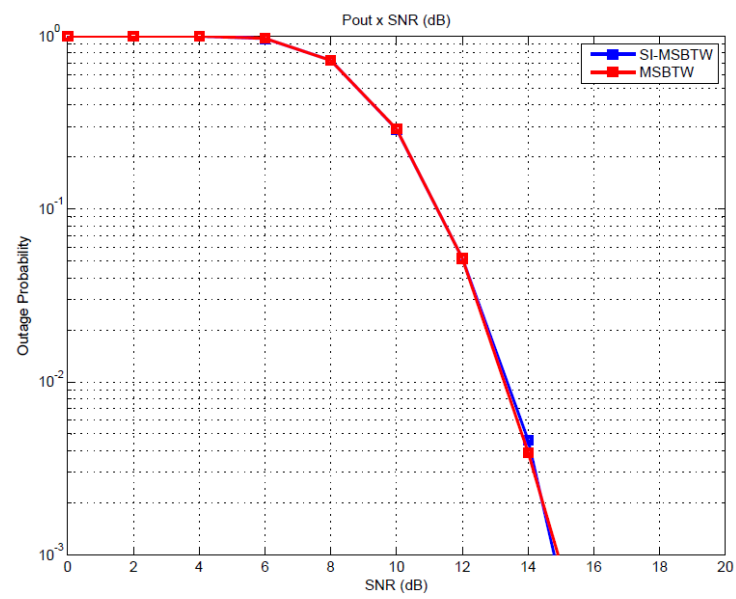

Fig. 7. Probabilidade de outage versus SNR para as técnicas MSBTW e SI-MSBTW.

proxima àquela da técnica MSBTW. Isto demonstra que a estimação não ruidosa do sinal transmitido pelo relay que a fonte realiza é bastante eficiente no que diz respeito à escolha da PAPR.

\section{CONClusões}

Neste artigo foram propostas duas novas técnicas de pareamento de subportadoras com redução da PAPR no relay em sistemas OFDM cooperativos utilizando o protocolo AF. As técnicas consistem em diminuir a PAPR no relay utilizando apenas o pareamento das subportadoras dos enlaces de comunicação, tendo como base a técnica de pareamento convencional BTW. Uma das técnicas propostas tem a vantagem de minimizar a PAPR dentro do conjunto de escolha, enquanto a outra tem a vantagem adicional de remover a complexidade do relay e deslocá-la para a fonte. Pode-se concluir com os resultados das simulações que as novas técnicas propostas apresentaram bom desempenho, visto que elas forneceram melhores PAPRs e SERs do que as outras técnicas testadas, com a mesma probabilidade de outage.

Em trabalhos futuros, pretende-se realizar uma análise de desempenho teórica detalhada das técnicas propostas, bem

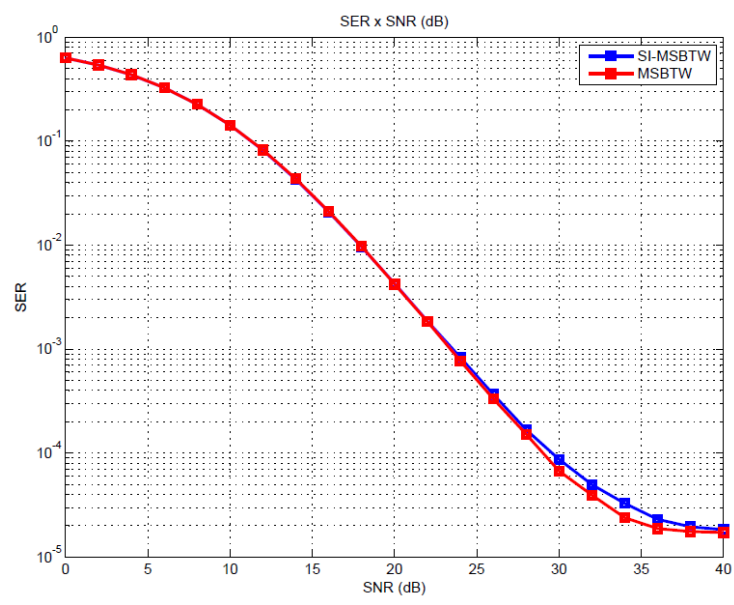

Fig. 8. SER versus SNR para as técnicas MSBTW e SI-MSBTW.

como generalizá-las para outros cenários, tal como utilizar o pareamento BTB e/ou outros protocolos de transmissão cooperativos, por exemplo.

\section{REFERÊNCIAS}

[1] K.J.R. Liu, A.K. Sadek, W. Su, and A. Kwasinski, Cooperative Communications and Networking. Cambridge University Press, 2009.

[2] E. L. Pinto, C. P. A. Albuquerque, "Técnica de Transmissão OFDM". Revista Científica Periódica - Telecomunicações. vol. 05, no. 01, 2002.

[3] Fernandes, C.A.R, Costa, D.B, Almeida, A.L.F, "Performance analysis of cooperative amplify-and-forward orthogonal frequency division multiplexing systems with power amplifier non-linearity", IET Communications. 2014

[4] M. Sabbaghian, "Reducing Required Power Back-Off of Nonlinear Amplifiers in Serial Modulation using SLM method", in Proceeding of the conference, Ottawa, Canada, 2005.

[5] S. G. Kang, J. G. Kim, and E. K. Joo, "A novel subblock partition scheme for partial transmit sequence OFDM", IEEE Transaction on Broadcast, vol. 45, pp. 333-338, 1999.

[6] L. Wang and J. Liu, "Cooperative PTS for PAPR reduction in MIMOOFDM", Electronics Letters, vol. 47, no. 5, 3rd March, 2011.

[7] B. Rani and G. Saini, "PAPR reduction using Cooperative PTS for Space Frequency Block Code MIMO-OFDM signal", 2nd IEEE International Conference on Parallel, Distributed and Grid Computing, 2012.

[8] G. P. Aquino e L. L. Mendes, "Comparação entre Técnicas de Redução da PAPR em Sinais OFDM", XXIX Simpósio Brasileiro de Telecomunicações - SBrT'11, Curitiba, PR, 2011.

[9] I. Hosseini, M.J. Omidi, K. Kasiri, A. Sadri, and P. G. Gulak, "PAPR Reduction in OFDM Systems Using Polynomial-Based Compressing and Iterative Expanding", IEEE International Conference on Acoustics Speech and Signal Processing Proceedings, 2006.

[10] M. M. Eddaghel and J. A. Chambers, "PAPR Reduction in Distributed Amplify-and-Forward Type Closed Loop Extended Orthogonal Space Frequency Block Coding with One-bit Group Feedback for Cooperative Communications", European Wireless 2012, Poznan, Poland, 2012.

[11] Y.J. Kim, U.K. Kwon, D.Y. Seol, and G.H. Im, "An Effective PAPR Reduction of SFBC-OFDM for Multinode Cooperative Transmission", IEEE Signal Processing Letters, vol. 16, no. 11, 2009.

[12] N. B. Harum , K. Yuda, and T. Ohtsuki, "PAPR Reduction of Amplifyand-Forward Relay OFDM System Using Subcarrier Pairing Method", IEEE 24th International Symposium on Personal, Indoor and Mobile Radio Communications: Mobile and Wireless Networks, 2013.

[13] E. Kocan, M.J Djurisic, D.S Michalopoulos, and G.K Karagiannidis, "Performance Evaluation of OFDM Amplify-and-Forward Relay System with Subcarrier Permutation", IEICE Trans. Commun. vol. E93-B, no. 5, pp. 1216-1223, 2010. 